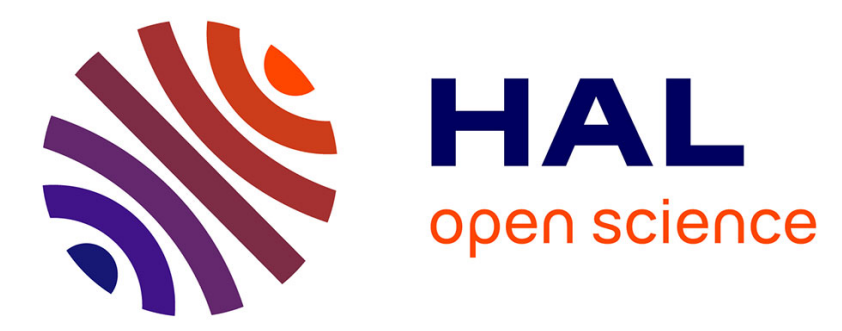

\title{
Planar Hall ring sensor for ultra-low magnetic moment sensing
}

Tran Quang Hung, Ferial Terki, Souleymanne Kamara, Kunwoo Kim, Salam Charar, Cheolgi Kim

\section{To cite this version:}

Tran Quang Hung, Ferial Terki, Souleymanne Kamara, Kunwoo Kim, Salam Charar, et al.. Planar Hall ring sensor for ultra-low magnetic moment sensing. Journal of Applied Physics, 2015, 117 (15), pp.154505. 10.1063/1.4918534 . hal-01203664

\section{HAL Id: hal-01203664 \\ https://hal.science/hal-01203664}

Submitted on 17 Mar 2021

HAL is a multi-disciplinary open access archive for the deposit and dissemination of scientific research documents, whether they are published or not. The documents may come from teaching and research institutions in France or abroad, or from public or private research centers.
L'archive ouverte pluridisciplinaire HAL, est destinée au dépôt et à la diffusion de documents scientifiques de niveau recherche, publiés ou non, émanant des établissements d'enseignement et de recherche français ou étrangers, des laboratoires publics ou privés.

\section{(ㄷ)(1)}

Distributed under a Creative Commons Attribution| 4.0 International License 


\title{
Planar Hall ring sensor for ultra-low magnetic moment sensing
}

\author{
Tran Quang Hung, ${ }^{1}$ Ferial Terki, ${ }^{2, a)}$ Souleymanne Kamara, ${ }^{2}$ Kunwoo Kim, ${ }^{3}$ Salam Charar, ${ }^{2}$ \\ and CheolGi Kim ${ }^{3, a)}$ \\ ${ }^{1}$ DTU-Food, Technical University of Denmark, Mфrkhфj Bygade 19, Sфborg DK-2860, Denmark \\ ${ }^{2}$ Institut Charles Gerhardt UMR 5253 CNRS-UM, Université de Montpellier, Montpellier 34000, France \\ ${ }^{3}$ Department of Emerging Materials Science, Daegu Gyeongbuk Institute of Science and Technology (DGIST), \\ Daegu 711-873, South Korea
}

The field sensitivity of a planar Hall effect (PHE) micro-ring type biosensor has been investigated as a function of magnetizing angle of the sensor material, for the sensing of low magnetic moment superparamagnetic labels. The field sensitivity is maximal at a magnetizing angle of $\alpha=20^{\circ}$. A t this optimized magnetizing angle, the field sensitivity of a PHE sensor is about 3.6 times higher than that measured at the conventional configuration, $\alpha=90^{\circ}$. This optimization enables the PHEring sensor to detect superparamagnetic biolabels with ultra-low magnetic moments down to $4 \times$ $10^{-13} \mathrm{emu}$.

\section{INTRODUCTION}

Magnetoresistive biosensors are an emerging field in healthcare because of their unique advantages: It is possible to create an inexpensive handheld point-of-care tool, which in a short time can easily be used to diagnose lifethreatening diseases or to monitor chronically ill patients. A magnetic biosensor detects biomarkers through the local stray field of their magnetic labels that are biologically bound to the sensor surface, under an external magnetic field. ${ }^{1}$ Because the sensor resolution is proportional to the detection capability of the magnetic moment, both the development of high magnetic moment labels such as $\mathrm{Fe}, \mathrm{CoFe}$, $\mathrm{Fe} @ \mathrm{Fe}_{3} \mathrm{O}_{4}$ nanoparticles, or nanoparticles of antiferromagnetic materials, ${ }^{2-6}$ and high field sensitive magnetoresistive sensors ${ }^{7}$ are highly desirable.

Among high field sensitive and low power consumption magnetoresistive biosensors, planar Hall effect (PHE) sensors have the advantages of high signal-to-noise ratio, small offset voltage, and linear response at small field. ${ }^{8}$ However, a typical crossed-junction PHE sensor has low field sensitivity. Thus, there has been extensive research to increase sensitivity by optimizing sensor materials (i.e., exchange bias bilayer thin films, ${ }^{9-12}$ spin valve thin films, ${ }^{13,14}$ trilayer thin films, ${ }^{15}$ and shape-induced anisotropy of a single ferromagnetic layer in an elliptically shaped element ${ }^{16,17}$ ) and sensor architectures (i.e., tilted cross junction, ${ }^{18}$ ring type,${ }^{19,20}$ and Wheatstone bridge type ${ }^{21}$ ).

For the detection of magnetic labels using PHE sensors, Ejsing et al. estimated that a single $2 \mu \mathrm{m}$ magnetic bead could give rise to a $10 \mu \mathrm{m} \times 10 \mu \mathrm{m}$ cross-junction sensor with a signal of $0.3 \mu \mathrm{V} .{ }^{9}$ Later on, we documented detection by a single magnetic bead by manipulating one magnetic bead ( $2.8 \mu \mathrm{m}$ on a $3 \mu \mathrm{m} \times 3 \mu \mathrm{m}$ cross-junction sensor); the bead decreased the voltage signal by about $1.1 \mu \mathrm{V} .{ }^{14}$ Thus, the detection capability of these sensor junctions was

\footnotetext{
a) Authors to whom correspondence should be addressed. Electronic addresses: ferial.terki@univ-montp2.fr and cgkim@dgist.ac.kr.
}

calculated to be about $10^{-10}-10^{-11}$ emu. Nevertheless, to detect such a small magnetic moment, the sensor junctions were fabricated in the size of a few micrometers, because the sensor signal is proportional to the ratio of the sensor area and the magnetic particle size. These small sensor junctions will produce large signal variance in the detection of microliter sample volumes. Consequently, these sensors are suitable for the detection or analysis of single cells only. In contrast, large-size sensors will provide a reproducible detection signal, but the sensitivity in detection of magnetic labels is low because of the compromise in their size. Therefore, improvement of sensitivity is desired.

In this paper, we optimized the field sensitivity of a large-scale 300- $\mu \mathrm{m}$ diameter PHE-ring sensor for the detection of low magnetic moments of magnetic labels. The field sensitivity of the sensor is optimized with respect to the angular dependence of the magnetic domain of the sensor material, a trilayer thin film. By combining these optimizations with a low-noise measurement method comprising continuous and alternative fields, the sensor can detect a very low magnetic moment of $10^{-14} \mathrm{emu}$.

\section{EXPERIMENTS}

\section{A. Sensor fabrication}

The PHE multi-ring sensors with five-ring architecture were fabricated by the lift-off of a trilayer structure of $\mathrm{Ta}(3) /$ $\mathrm{NiFe}(10) / \mathrm{Cu}(0.1) / \mathrm{IrMn}(10) / \mathrm{Ta}(3)(\mathrm{nm})$ on a $1.5-\mu \mathrm{m}$ photoresist layer. The outer radii of the ring elements were $r_{\mathrm{i}}=150$, $120,90,60$, and $30 \mu \mathrm{m}$, and the width of the rings was $5 \mu \mathrm{m}$. The easy axis of the sensors was formed by an in-plane DC magnetic field of $20 \mathrm{mT}$ applied during deposition of the trilayer thin films. The sensors were connected with the external circuitry by $100-\mathrm{nm}$ Au electrodes. To prevent electrical leakage of the sensor from liquid solution, an insulating bilayer thin film of $200 \mathrm{~nm} \mathrm{Si} \mathrm{N}_{4} / \mathrm{SiO}_{2}$ was deposited on top of the sensors for passivation. A scanning electron microscopy (SEM) image of a PHE-ring sensor with defined parameters is shown in Fig. 1(a). 

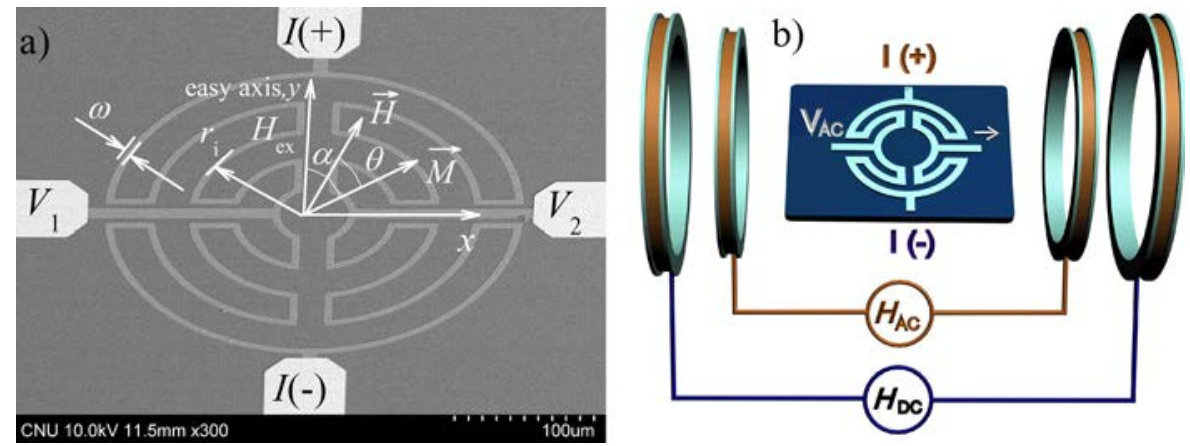

FIG. 1. (a) SEM image of a multi-ring sensor with radius element $r_{\mathrm{i}}=120 \mu \mathrm{m}$, arm width $(\omega)$, and sensor electrodes for applying current $(I(+), I(-))$ and measurement of PHE voltage $\left(V_{1}, V_{2}\right)$. The magnetization $(M)$ and applied magnetic field $(H)$ directions, the applied field angle from the easy axis $(\alpha)$, and the angle between the magnetization and applied field directions $(\theta)$ are schematically drawn. (b) Measurement setup combining AC and DC field components for sensor characterization and for probing the signal of superparamagnetic labels on the PHE sensor.

\section{B. Sensor characterization}

The magnetic properties of the fabricated sensors were investigated by a vibrating sample magnetometer with exchange bias field $H_{\mathrm{ex}}=4.9 \mathrm{kA} / \mathrm{m}$, coercivity field $H_{\mathrm{k}}=477 \mathrm{~A} / \mathrm{m}$, and saturation magnetization $M_{\mathrm{s}}=400 \mathrm{~m}$ $\mathrm{emu} \cdot \mathrm{cm}^{-3}$. To avoid sensor frequency noise and offset voltage, we used mixed fields of continuous and alternative components (AC and DC) applied to the sensor with the same direction, as schematically shown in Fig. 1(b). The $113 \mathrm{~Hz}$ $\mathrm{AC}$ field was chosen to minimize the $1 / \mathrm{f}$ noise of the sensor. At this frequency, only white noise contributes to the noise spectrum of the sensor, because the $1 / \mathrm{f}$ corner of the noise spectra was found to be about $13 \mathrm{~Hz}$. The PHE voltage signals were measured by means of a highly sensitive RS830 digital lock-in amplifier.

\section{RESULTS AND DISCUSSION}

Typically, when the magnetic field is applied perpendicular to the easy axis of the thin film, the magnetic domain rotates towards the applied magnetic field coherently as a result of its strong magnetic energy. However, when the field is parallel to the easy axis, the domain switches easily, following the magnetic field direction through the domain wall motion. In the following, we investigate the angular dependence of the PHE voltage ( $\left.V_{\mathrm{PHE}}\right)$ profiles to optimize the field sensitivity of a PHE-ring sensor. The voltage profile with the field applied parallel to the easy axis of the magnetic domain is shown in Fig. 2(a). Here, an hysteretic profile appears around the exchange bias field because the magnetic domain does not rotate coherently with the magnetic field when the magnetic field is compatible with the unidirectional field, $H_{\mathrm{ex}} \cdot{ }^{22}$ This is caused by a change in the minimum energy of the domain, which is described by the Stoner-Wohlfarth energy model ${ }^{23}$ for domain rotation. This hysteresis relates to the domain wall motion at low magnetizing angle and would be reduced when increasing the applied field angle, $\alpha$, from the easy axis of the sensor. ${ }^{24,25}$ The inset in Fig. 2(a) shows the calculated hysteretic width of a PHE voltage profile as a function of $\alpha$. As per our calculation, the hysteresis disappears when $\alpha \geq 4^{\circ}$. Therefore, the external magnetic field should be applied to the sensor with an angle higher than this critical angle, $\alpha_{\mathrm{c}}$. However, this critical angle is dependent on the uniaxial field, $H_{\mathrm{k}}$, of the multilayers.

When $\alpha \geq 4^{\circ}$, the voltage profiles are not hysteretic and they show asymmetric behavior. The PHE voltage profiles of the investigated ring sensor with different magnetizing angles are illustrated in Fig. 2(b). The calculated profiles for these magnetizing angles are plotted together as solid lines. To calculate the PHE voltage profiles, the equation system of minimum energy of a single domain (Stoner-Wohlfarth energy) and the planar Hall voltage deduced from Ohm's law were used. The details of this calculation have been discussed elsewhere. ${ }^{20}$ In this calculation, we used the
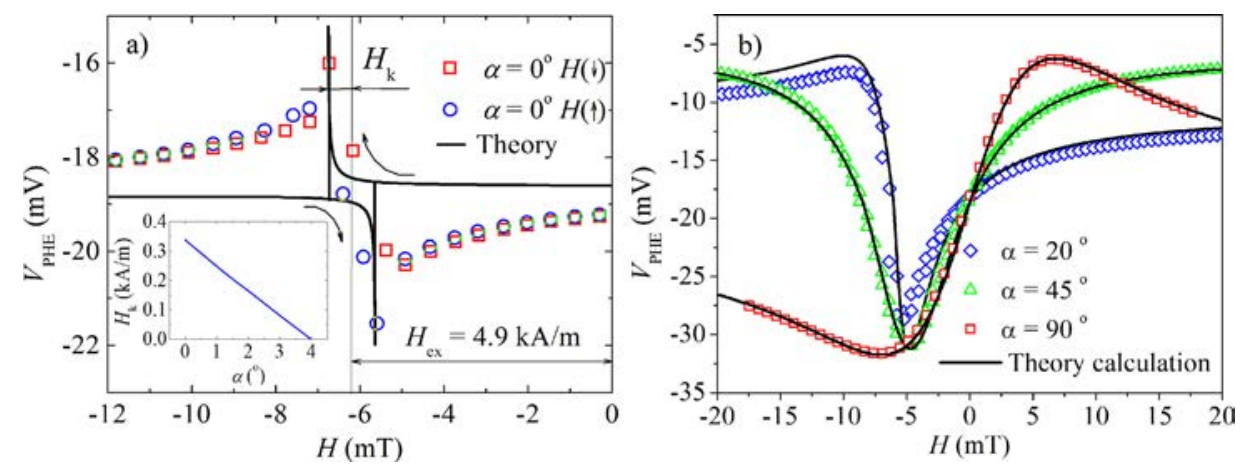

FIG. 2. (a) Calculated PHE voltage profile with respect to the applied field of a multi-ring sensor at $\alpha=0^{\circ}$ for domain switching (solid black line); arrows indicate the direction of the field variation. Measurements are plotted as open squares (decreasing field) and open circles (increasing field). The inset shows the calculation of the hysteretic width of the PHE voltage profile at small angle $\alpha$. (b) Calculated and measured PHE voltage profiles at $\alpha=20^{\circ}, 45^{\circ}$, and $90^{\circ}$. A constant voltage of $-18.9 \mathrm{mV}$ was added to the calculated data to account for the offset voltage of the measurements. 

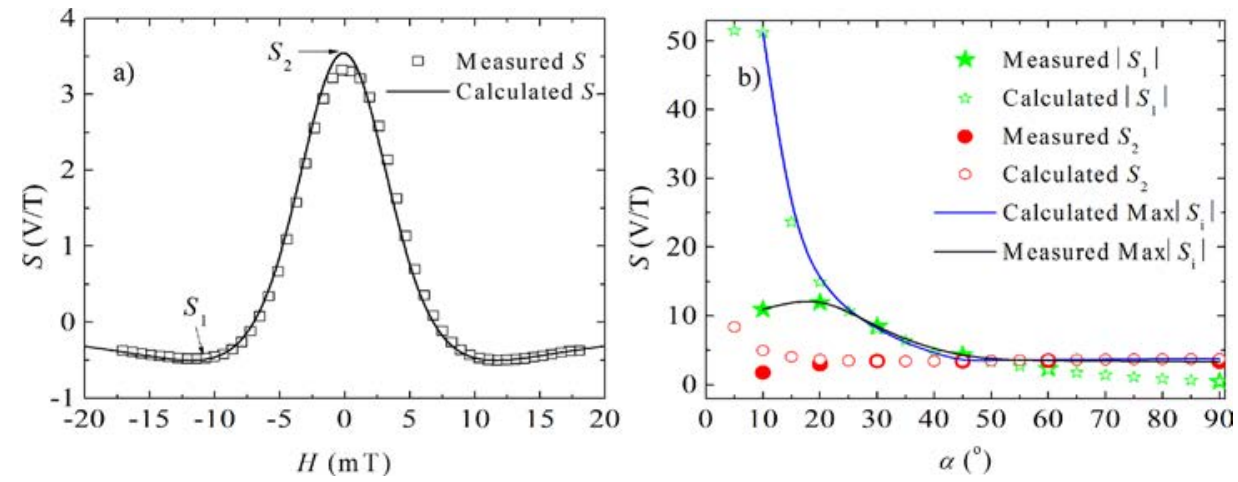

FIG. 3. (a) Field sensitivity of a PHE sensor as a function of applied magnetic field for $\alpha=90^{\circ}$, plotting measured data (open squares), and calculations (solid line). (b) Field sensitivities $S_{1}$ (calculations as open and measurements as solid stars) and $S_{2}$ (calculations as open and measurements as solid circles). The calculation values are for each $5^{\circ}$ step of $\alpha$, and the measured field sensitivities are for $\alpha=10^{\circ}, 20^{\circ}, 30^{\circ}, 45^{\circ}, 60^{\circ}$, and $90^{\circ}$. The calculated and measured maximal field sensitivities as a function of applied field angle are plotted as blue and black solid lines, respectively.

measured parameters $H_{\mathrm{ex}}=-4.9 \mathrm{kA} / \mathrm{m}, H_{\mathrm{k}}=-477 \mathrm{~A} / \mathrm{m}$, $M_{\mathrm{s}}=400 \mathrm{~m} \mathrm{emu} \mathrm{cm}^{-3}$, current $I=1 \mathrm{~mA}$, amplitude of voltage profiles $V_{\mathrm{o}}=12.6 \mathrm{mV}$, and drift voltage of $-18.9 \mathrm{mV}$. The angular dependence that the PHE voltage profile exhibits in the ring sensor is similar to that in the cross-junction sensor; ${ }^{25}$ thus, the single-domain model used in these calculations is convincing.

To find the best performance of the sensor, the field sensitivity, $S$, of a PHE-ring sensor $\left(S_{\mathrm{PHE}}=\frac{\partial V_{\mathrm{PHE}}}{\partial H}\right)$ was investigated as a function of applied field angle $\alpha$. The dependence of the field sensitivity with respect to the magnetizing angle $\alpha$ can be calculated from the single domain model and expressed as follows: ${ }^{26}$

$$
S_{\text {PHE }}=\frac{r I \Delta \rho}{2 \omega t} \frac{\cos 2 \theta \sin (\alpha-\theta)}{H_{\mathrm{k}} \cos 2 \theta+H_{\mathrm{ex}} \cos \theta+H \cos (\alpha-\theta)} .
$$

Here, $r$ and $\omega$ are the radii and width of the ring element, respectively; and $\theta, \Delta \rho$, and $t$ are the angle between the easy axis of the magnetic domain and applied field, anisotropic resistivity difference, and thin film thickness, respectively. For the investigated sensor, $H_{\mathrm{k}}$ and $H_{\mathrm{ex}}$ are constant, thus the maximal $S_{\mathrm{PHE}}$ at each angle $\alpha$ can be calculated over the interval of the applied fields $(H)$. The calculated and measured field sensitivities as a function of magnetic field at $\alpha=90^{\circ}$ are shown in Fig. 3(a). In the field range from 0 to $-20 \mathrm{mT}$, there are two extrema, $S_{1}$ and $S_{2}$, in the field sensitivity profiles. The calculated and measured $S_{1}$ and $S_{2}$ are plotted in Fig. 3(b) for $\alpha_{\mathrm{c}}<\alpha<90^{\circ}$.

In Fig. 3, only absolute values are considered because negative and positive values correspond to increasing and decreasing PHE voltage. Figure 3(b) shows that $\left|S_{1}\right|$ increases and $\left|S_{2}\right|$ decreases with angle $\alpha$, and they cross at $45^{\circ}$. Thus, the calculated and measured maximal field sensitivities as a function of $\alpha$ are the values of $\max \left[\left|S_{1}\right|,\left|S_{2}\right|\right]$, plotted as blue and black solid lines. These values decrease slightly when decreasing $\alpha$ from $90^{\circ}$ to $45^{\circ}$ (behavior of $\left|S_{2}\right|$ ), then increase with further decreasing $\alpha$ (behavior of $\left.\left|S_{1}\right|\right)$. However, there are differences between the measured and calculated field sensitivity values at low magnetizing angles $\left(<20^{\circ}\right)$. In the calculation, the field sensitivity reaches a maximum at $\alpha=10^{\circ}$, whereas the measured value is maximal at $\alpha=20^{\circ}$. In comparison, the calculated maximal field sensitivity is about 14 times greater than that determined with the conventional measurement configuration $\left(\alpha=90^{\circ}\right)$, whereas this factor is only about four times greater in the measured data. These differences may be because domain rotation is assumed for all magnetizing angles in the calculations, but the domain wall motion is involved in the sensor at low magnetizing angles, $\alpha<20^{\circ}$.

In the detection of superparamagnetic labels using particles or beads for biochip applications, therefore, the magnetizing angle of the sensor must be carefully adjusted to achieve maximal sensitivity for the detection of magnetic labels. Magnetic particles appearing on the PHE multi-ring sensor will decrease the PHE voltage signal, $\Delta V$, which is proportional to the production of the field sensitivity of the sensor and the applied field as follows: ${ }^{26}$

$$
\Delta V \sim-\left[\sum_{i=1}^{n} \frac{R^{3} \chi N}{3 z_{\mathrm{o}}^{3}\left(1+\frac{r_{i}^{2}}{z_{\mathrm{o}}^{2}}\right)^{3 / 2}}\right] H_{\mathrm{AC}} \cdot S .
$$

Here, $\chi N, R, r_{\mathrm{i}}$, and $z_{\mathrm{o}}$ are volume susceptibility, number and mean radius of the magnetic particles, radii of the ring elements, and distance from the particles to the sensor surface, as schematically illustrated in Fig. 4(a).

To optimize sensitivity for the detection of magnetic labels at each angle $\alpha$, we fixed the AC field at $H_{\mathrm{p}-\mathrm{p}}=1 \mathrm{mT}$, $f=113 \mathrm{~Hz}$ for low noise detection, fixed $H_{\mathrm{AC}}$ in Eq. (2), and selected the DC field to obtain the maximal field sensitivity. For the investigated sensor, the DC field was about $-7 \mathrm{mT}$ for $\alpha$ in the range from $10^{\circ}$ to $30^{\circ}$, and the DC fields were -1.8 and $-3 \mathrm{mT}$ for $\alpha=60^{\circ}$ and $90^{\circ}$, respectively. For these optimizations, we monitored the PHE voltage signal when injecting a $2 \mu \mathrm{l}$ droplet of magnetic solution on the sensor surface, which contains $0.1 \% \mathrm{M}-280$ streptavidin magnetic beads $^{27}$ (Life Technologies ${ }^{\mathrm{TM}}$ ) in $\mathrm{pH} 7.4$ phosphate buffered saline (PBS) buffer. $\Delta V$ was recorded in three 3-min cycles; the average $\Delta V$ for the three cycles at magnetizing angles $\alpha=10^{\circ}, 20^{\circ}, 30^{\circ}, 60^{\circ}$, and $90^{\circ}$ are plotted in Fig. 4(b). At the maximum field sensitivity configuration $\left(\alpha=20^{\circ}\right)$, the voltage change of the sensor was about 3.6 times higher than that measured at $\alpha=90^{\circ}$. The maximal $\Delta V$ was obtained at 

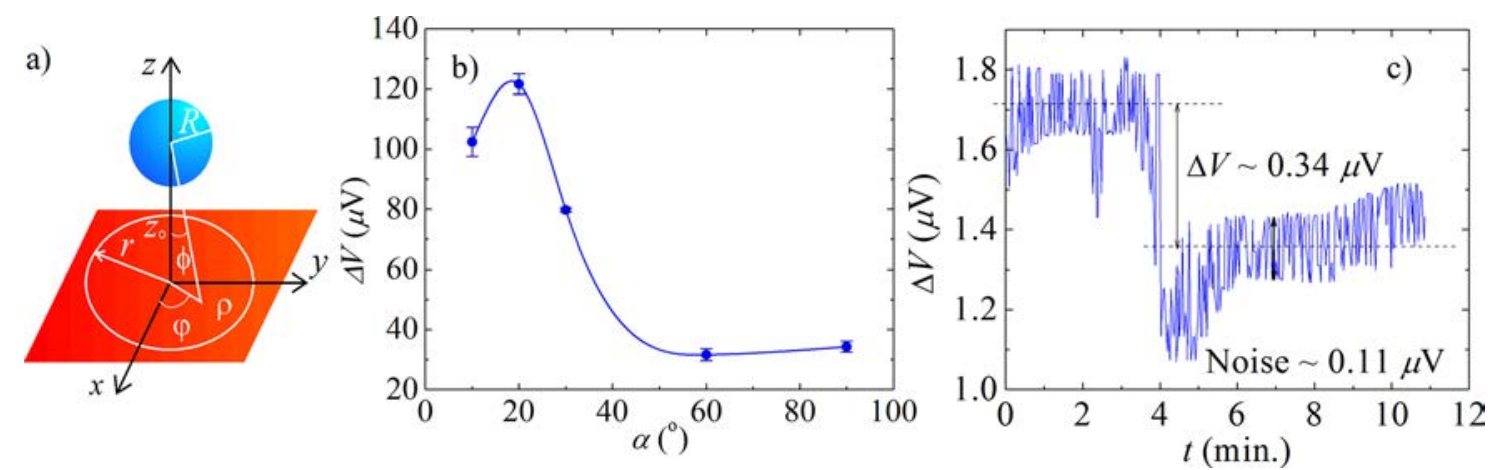

FIG. 4. (a) Schematic of a magnetic particle on a ring sensor with defined parameters in the calculation appearing in Eq. (2). (b) Detection voltage of the PHE multi-ring sensor responding to a $2 \mu \mathrm{l}$ droplet of magnetic bead solution ( $0.1 \% \mathrm{M}-280$ streptavidin magnetic bead in pH $7.4 \mathrm{PBS}$ buffer) at different magnetizing angles; the solid line represents its trend. (c) Real-time monitoring of $1 \mu$ superparamagnetic nanoparticles (1:10 7 dilution of stock solution in PBS buffer) by the PHE-ring sensor operated at the highest sensitivity configuration, $\alpha=20^{\circ}$.

$\alpha=20^{\circ}$, demonstrating that control of magnetization in the sensor material is very important for low magnetic signal detection.

To quantify the resolution of the sensor detection, we fixed the sensor at the highest field sensitivity configuration and measured the voltage signal of $1 \mu \mathrm{l}$ droplets of fluidMAG-streptavidin nanoparticles ${ }^{28}\left(\mathrm{Fe}_{3} \mathrm{O}_{4}\right.$, mean size of $\sim 100 \mathrm{~nm}$ ) at different dilutions in PBS buffer. The real-time measurement of the lowest concentration of $c a$. 1800 nanoparticles in a $1 \mu \mathrm{l}$ droplet $\left(1: 10^{7}\right.$ dilution ratio from stock solution) is shown in Fig. 4(c). In this case, the sensor reduces a voltage of $0.34 \mu \mathrm{V}$ with a signal-to-noise ratio of about 3 . The decrease in the signal is related to the opposite stray field direction to the applied field for superparamagnetic nanoparticles. With the estimated susceptibility value of $\mathrm{Fe}_{3} \mathrm{O}_{4}$ particles, $\chi_{\mathrm{V}}=5$ (International system of units) and the mean radius of the particles $(r=50 \mathrm{~nm})$, the magnetic moment of the droplet can be calculated to be $c a$. $4 \times 10^{-13}$ emu using equation $m=n V_{\text {particle }} \chi_{V} H(n=\sim 1800$ nanoparticles, $H=10^{-3} \mathrm{~T}$, and $V_{\text {particle }}=4 \pi r^{3} / 3$ ). This is the best magnetic moment resolution among sensors of the same size operating at room temperature. Note that the magnetic moment of this droplet cannot be detected using a state-ofthe-art, highly sensitive, superconducting quantum interference device (SQUID) system; its sensitivity is about $10^{-10} \mathrm{emu}$. For sensitivity to low magnetic moments, some nano-sized magnetoresistive sensors were investigated for the detection of single nanoparticles. However, for bioapplications, sensors with large dimensions are desirable for the detection of large sample volumes. In this respect, the high sensitivity of the few hundred-micrometer-sized sensors achieved in this work is highly important.

\section{CONCLUSIONS}

In summary, we have demonstrated by both experiment and calculations that the field sensitivity of a PHE multi-ring sensor is strongly dependent on the magnetizing angle of the thin film. The maximum field sensitivity is obtained when the magnetizing angle of the sensor is $20^{\circ}$. Further, using a measurement setup combining AC and DC fields enables us to probe low concentrations of injected superparamagnetic labels. In comparison with the conventional measurement configuration $\left(\alpha=90^{\circ}\right)$, this optimized measurement enhances both the field sensitivity and sensitivity for the detection of magnetic labels of a PHE sensor by a factor of about 3.6. Moreover, the combination of the optimized magnetizing angle of the sensor in this work, the recently developed sensor material (trilayer thin film) and sensor architecture (multi-ring PHE sensor), enables us to detect magnetic moments as low as $4 \times 10^{-13} \mathrm{emu}$, at least three orders of magnitude lower than the detection resolution reported earlier in several PHE cross-junction sensors. This novel optimization method could be a paradigm to increase the sensitivity of any PHE sensor such as a cross-junction, elliptically shaped, PHE bridge junction, or PHE-ring junction sensors.

\section{ACKNOWLEDGMENTS}

This work was partially supported by a 2014 DGIST start-up fund. The authors F. Terki, T. Q. Hung, and S. Kamara are grateful for the financial support from the project EADS Foundation, the Labex-NUMEV, and the ANR-10-LABX-05-01 through the Labex-CHEMISYST.

\footnotetext{
${ }^{1}$ A. Sandhu, Nat. Nanotechnol. 2, 746 (2007).

${ }^{2}$ D. L. Huber, Small 1, 482 (2005).

${ }^{3}$ H. Kura, M. Takahashi, and T. Ogawa, J. Phys. Chem. C 114, 5835 (2010)

${ }^{4}$ C. Wang, S. Peng, L.-M. Lacroix, and S. Sun, Nano Res. 2, 380 (2009).

${ }^{5}$ A.-H. Lu, E. L. Salabas, and F. Schüth, Angew. Chem., Int. Ed. Engl. 46, 1222 (2007).

${ }^{6}$ W. Hu, R. J. Wilson, A. Koh, A. Fu, A. Z. Faranesh, C. M. Earhart, S. J. Osterfeld, S.-J. Han, L. Xu, S. Guccione, R. Sinclair, and S. X. Wang, Adv. Mater. 20, 1479 (2008).

${ }^{7}$ P. P. Freitas, H. A. Ferreira, D. L. Graham, L. A. Clarke, M. D. Amaral, V. Martins, L. Fonseca, and J. S. Cabral, in Magnetoelectronics, edited by M. Johnson (ScienceDirect, 2004), pp. 331-386.

${ }^{8}$ T. Q. Hung, D.-Y. Kim, B. P. Rao, and C. G. Kim, in State of the Art in Biosensors - General Aspects, edited by T. Rinken (Intech, 2013), pp. 197-239.

${ }^{9}$ L. Ejsing, M. F. Hansen, A. K. Menon, H. A. Ferreira, D. L. Graham, and P. P. Freitas, Appl. Phys. Lett. 84, 4729 (2004).

${ }^{10}$ A. Schuhl, F. N. Van Dau, and J. R. Childress, Appl. Phys. Lett. 66, 2751 (1995)

${ }^{11}$ B. D. Tu, T. Q. Hung, N. T. Thanh, T. M. Danh, N. H. Duc, and C. G. Kim, J. Appl. Phys. 104, 074701 (2008).

${ }^{12}$ F. Qejvanaj, M. Zubair, A. Persson, S. M. Mohseni, V. Fallahi, S. R. Sani, S. Chung, T. Le, F. Magnusson, and J. Akerman, IEEE Trans. Magn. 50, 4006104 (2014)
} 
${ }^{13}$ B. D. Tu, L. V. Cuong, T. Q. Hung, D. T. H. Giang, T. M. Danh, N. H. Duc, and C. G. Kim, IEEE Trans. Magn. 45, 2378 (2009).

${ }^{14}$ T. Q. Hung, S. Oh, J.-R. Jeong, and C. Kim, Sens. Actuators, A 157, 42 (2010).

${ }^{15}$ T. Q. Hung, S. Oh, B. Sinha, J.-R. Jeong, D.-Y. Kim, and C. Kim, J. Appl. Phys. 107, 09E715 (2010).

${ }^{16}$ I. Genish, Y. Shperber, N. Naftalis, G. Salitra, D. Aurbach, and L. Klein, J. Appl. Phys. 107, 09E716 (2010).

${ }^{17}$ V. Mor, M. Schultz, O. Sinwani, A. Grosz, E. Paperno, and L. Klein, J. Appl. Phys. 111, 07E519 (2012).

${ }^{18}$ T. Q. Hung, J.-R. Jeong, D.-Y. Kim, N. H. Duc, and C. Kim, J. Phys. D: Appl. Phys. 42, 055007 (2009).

${ }^{19}$ S. Oh, P. B. Patil, T. Q. Hung, B. Lim, M. Takahashi, D. Y. Kim, and C. Kim, Solid State Commun. 151, 1248 (2011).

${ }^{20}$ B. Sinha, T. Q. Hung, T. S. Ramulu, S. Oh, K. Kim, D.-Y. Kim, F. Terki, and C. Kim, J. Appl. Phys. 113, 063903 (2013).
${ }^{21}$ A. D. Henriksen, B. T. Dalslet, D. H. Skieller, K. H. Lee, F. Okkels, and M. F. Hansen, Appl. Phys. Lett. 97, 013507 (2010).

${ }^{22}$ J. Ye, W. He, Q. Wu, H.-L. Liu, X.-Q. Zhang, Z.-Y. Chen, and Z.-H. Cheng, Sci. Rep. 3, 2148 (2013).

${ }^{23}$ E. C. Stoner and E. P. Wohlfarth, IEEE Trans. Magn. 27, 3475 (1991).

${ }^{24}$ T. Ambrose, R. Sommer, and C. Chien, Phys. Rev. B 56, 83 (1997).

${ }^{25}$ N. T. Thanh, M. G. Chun, J. Schmalhorst, G. Reiss, K. Y. Kim, and C. G. Kim, J. Magn. Magn. Mater. 304, e84 (2006).

${ }^{26}$ T. Q. Hung, F. Terki, S. Kamara, M. Dehbaoui, S. Charar, B. Sinha, C. Kim, P. Gandit, I. A. Gural'Skiy, G. Molnar, L. Salmon, H. J. Shepherd, and A. Bousseksou, Angew. Chem., Int. Ed. 52, 1185 (2013).

${ }^{27} \mathrm{See} \quad$ http://tools.lifetechnologies.com/content/sfs/manuals/dynabeads_ m280SAv_man.pdf for information on Dynabeads M-280 Streptavidin.

${ }^{28}$ See http://www.chemicell.com/products/nanoparticles/docs/PI_fluidMAGStreptavidin_4205.pdf for information on FluidMAG-Streptavidin particles. 